\title{
Emergent Behavior in Flocks
}

\author{
Felipe Cucker * \\ Department of Mathematics \\ City University of Hong Kong \\ 83 Tat Chee Avenue, Kowloon \\ HONG KONG \\ e-mail: macucker@math.cityu.edu.hk \\ Steve Smale ${ }^{\dagger}$ \\ Toyota Technological Institute at Chicago \\ 1427 East 60th Street, Chicago, IL 60637 \\ U.S.A. \\ e-mail: smale@math. berkeley.edu
}

July 13, 2005

\section{PRELIMINARY VERSION.}

\section{Introduction}

As a motivating example we consider a population, say of birds or fish, whose members are moving in $\mathbb{R}^{3}$. It has been observed that under some initial conditions, for example on their positions and velocities, the state of the flock converges to one in which all birds fly with the same velocity. A goal of this paper is to provide some justification of this observation. To do so, we will postulate a model for the evolution of the flock and exhibit conditions on the initial state under which a convergence as above is established. In case these conditions are not satisfied, dispersion of the flock may occur.

There has been a large amount of literature on flocking, herding and schooling. Much of it is descriptive, most of the remaining proposes models, which are then studied via computer simulations, e.g., $[3,7]$. A starting point for this paper is the model proposed in the latter of these references which, for convenience, we will call

\footnotetext{
${ }^{*}$ Partially funded by a grant from the Research Grants Council of the Hong Kong SAR (project number CityU 1085/02P). Thanks are due to the Toyota Technological Institute at Chicago for a very pleasant stay there in May 2005.

${ }^{\dagger}$ Partially supported by an NSF grant. This author also wants to thank City University of Hong Kong for inviting me for a month during June 2005.
} 
Vicsek's model. Its analytic behavior was subsequently studied in [4] and this paper, brought to our attention by Ali Jadbabaie, has been helpful for us.

Vicsek's model is motivated by the idea that bird $i$ adjusts its velocity towards the average of its neighbors' velocities. With (our first modification) $\mathbb{R}^{2}$ replaced by Euclidean space $\mathbb{E}^{3}$ and the heading $\theta$ replaced by the velocity $v$. The model is

$$
\begin{aligned}
& x_{i}(t+1)=x_{i}(t)+v_{i}(t) \\
& v_{i}(t+1)=\frac{1}{n_{i}(t)} \sum_{j \in \mathcal{N}_{i}(t)} v_{j}(t) .
\end{aligned}
$$

where $x_{i}, v_{i} \in \mathbb{E}^{3}$ for $i=1, \ldots, k$ and time $t=0,1,2 \ldots$ Here $\mathcal{N}_{i}(t)=\{j \leq k \mid$ $\left.\left\|x_{i}(t)-x_{j}(t)\right\| \leq r\right\}$ and $n_{i}(t)=\# \mathcal{N}_{i}(t)$ for some $r>0$.

Let $A_{x}$ be the $k \times k$ binary matrix given by

$$
a_{i j}= \begin{cases}1 & \text { if }\left\|x_{i}(t)-x_{j}(t)\right\| \leq r \\ 0 & \text { otherwise }\end{cases}
$$

Denoting by $A_{i}$ the $i$ th row of $A_{x}$ and by $\mathbf{e}_{i}$ the vector $(0, \ldots, 0,1,0, \ldots, 0)$ with the 1 in the $i$ th place, we have

$$
v_{i}(t+1)-v_{i}(t)=\left(\frac{1}{n_{i}(t)} A_{i}-\mathbf{e}_{i}\right) v(t)=\frac{1}{n_{i}(t)}\left[A_{i}-n_{i} \mathbf{e}_{i}\right] v(t) .
$$

The last two expressions should be understood as linear combinations of elements in $\mathbb{E}^{3}$. Extending this notation to matrix form,

$$
v(t+1)-v(t)=-D_{x}^{-1} L_{x} v(t)
$$

where $D_{x}$ is the $k \times k$ diagonal matrix whose $i$ th diagonal entry is $\sum_{j \leq k} a_{i j}$ and $L_{x}$ is the matrix whose $i$ th row is $A_{i}-n_{i} \mathbf{e}_{i}$, that is,

$$
L_{x}=D_{x}-A_{x} .
$$

We found it convenient to modify (2) by scaling $L_{x}$ in a slightly different way, namely,

$$
\begin{aligned}
x(t+1) & =x(t)+\Delta t v(t) \\
v(t+1) & =\left(\operatorname{Id}-\frac{L_{x}}{\left\|L_{x}\right\|}\right) v(t) .
\end{aligned}
$$

Here $\left\|L_{x}\right\|$ is the operator norm of $L_{x}$ (with respect to the norm in $\left(\mathbb{E}^{3}\right)^{k}$ induced by the norm of $\mathbb{E}^{3}$ ).

Our third modification proceeds as follows. It is reasonable to assume that birds influence each other as a function of their distance. We give form to this assumption 
via a non-increasing function $\eta: \mathbb{R}_{+} \rightarrow \mathbb{R}_{+}$such that the adjacency matrix $A_{x}$ has entries

$$
a_{i j}=\eta\left(\left\|x_{i}-x_{j}\right\|^{2}\right) .
$$

In this paper we will take, for some fixed $K, \sigma>0$ and $\beta \geq 0$,

$$
\eta(y)=\frac{K}{\left(\sigma^{2}+y\right)^{\beta}} .
$$

Vicsek's adjacency matrix is also of this form where, for some $r>0$,

$$
\eta(y)= \begin{cases}1 & \text { if } y \leq r^{2} \\ 0 & \text { otherwise }\end{cases}
$$

Note that, in contrast with the abrupt behavior of this last function, the function in (6) decreases continuously with $y$ and the rate of decay is given by $\beta>0$.

We also consider evolution for continuous time. The corresponding model can be given by the system of differential equations

$$
\begin{aligned}
x^{\prime} & =v \\
v^{\prime} & =-L_{x} v .
\end{aligned}
$$

Our first two main results give conditions to ensure that the birds' velocities converge to a common one and the distance between birds remain bounded for both continuous and discrete time. They can be stated as follows (more precise statements are in Theorems 2 and 3 below).

Theorem 1 Let $(x(t), v(t))$ be a solution of (4) with initial conditions $x(0)=x_{0}$ and $v(0)=v_{0}$. If $\beta<1 / 2$ then, when $t \rightarrow \infty$ the velocities $v_{i}(t)$ tend to a common limit $\widehat{v} \in \mathbb{E}^{3}$ and the vectors $x_{i}-x_{j}$ tend to a limit vector $\widehat{x_{i j}}$, for all $i, j \leq k$. The same happens if $\beta \geq 1 / 2$ provided the initial values $x_{0}$ and $v_{0}$ satisfy a given, explicit, relation.

The same holds for a solution of (7).

\section{Some preliminaries}

Given a nonnegative, symmetric, $k \times k$ matrix $A$ the Laplacian $L$ of $A$ is defined to be

$$
L=D-A
$$

where $D=\operatorname{diag}\left(d_{1}, \ldots, d_{k}\right)$ and $d_{\ell}=\sum_{j=1}^{k} a_{\ell j}$. Some features of $L$ are immediate. It is symmetric and it does not depend on the diagonal entries of $A$. The Laplacian as just defined has its origins in graph theory where the matrix $A$ is the adjacency matrix of a graph $G$ and many of the properties of $G$ can be read out from $L$ (see [6]).

The matrix $L_{x}$ in (4) and (7) is thus the Laplacian of $A_{x}$. It acts on $\left(\mathbb{E}^{3}\right)^{k}$ and satisfies the following: 
(a) For all $v \in \mathbb{E}^{3}, L_{x}(v, \ldots, v)=0$.

(b) If $\lambda_{1}, \ldots, \lambda_{k}$ are the eigenvalues of $L_{x}$ then

$$
0=\lambda_{1} \leq \lambda_{2} \leq \ldots \leq \lambda_{k}=\left\|L_{x}\right\|
$$

(c) For all $v \in\left(\mathbb{E}^{3}\right)^{k}$,

$$
\left\langle L_{x} v, v\right\rangle=\sum_{i, j=1}^{k} a_{i j}\left\|v_{i}-v_{j}\right\|^{2} .
$$

Note that (b) implies $L_{x}$ is positive semidefinite.

The quantity $E_{x}(v)=\sum_{i, j=1}^{k} a_{i j}\left\|v_{i}-v_{j}\right\|^{2}$ is the energy of the flock (at a position $x \in\left(\mathbb{E}^{3}\right)^{k}$ and a velocity $\left.v \in\left(\mathbb{E}^{3}\right)^{k}\right)$. Note that $E_{x}(v)=0$ when all birds are flying with the same velocity. That is, they fly with the same heading and at the same speed.

The matrix $\operatorname{Id}-\frac{1}{\left\|L_{x}\right\|} L_{x}$ in $(4)$ acts on $\left(\mathbb{E}^{3}\right)^{k}$. The fixed points for this action are easily characterized.

Proposition 1 Let $v \in\left(\mathbb{E}^{3}\right)^{k}$. The following are equivalent:

(1) $v$ is a fixed point (i.e., $\left.\left(\operatorname{Id}-\frac{1}{\left\|L_{x}\right\|} L_{x}\right) v=v\right)$.

(2) $L_{x}(v)=0$.

(3) $E_{x}(v)=0$.

Proof. The equivalence between (1) and (2) is obvious. The implication (2) $\Longrightarrow$ (3) is trivial. Finally, note that (3) implies that $v_{i}=v_{j}$ for all $i \neq j$ and this, together with (a) above, implies (2).

The second eigenvalue $\lambda_{2}$ of $L_{x}$ is called the Fiedler number of $A_{x}$. We denote the Fiedler number of $A_{x}$ by $\phi_{x}$.

Remark 1 One difference between Vicsek's model and (4) lies in the way in which $L_{x}$ is scaled. In (2) the scaling used is $D_{x}^{-1} L_{x}$. The product matrix $H=D_{x}^{-1} L_{x}$ satisfies that, for all $i \leq k,\left\|H_{i}\right\|_{\infty}=1$. Also, the product matrix $S=D_{x}^{-1} A_{x}$ is stochastic (i.e., nonnegative and such that $\left\|S_{i}\right\|_{1}=1$ ). We note, however, that it destroys the symmetry of both $A_{x}$ and $L_{x}$.

The scaling in (4) considers instead $\frac{L_{x}}{\left\|L_{x}\right\|}$. This does not lead to stochasticity but preserves symmetry.

We end these preliminaries introducing some concepts which will be useful in this paper.

Let $\Delta$ be the diagonal of $\left(\mathbb{E}^{3}\right)^{k}$, i.e.,

$$
\Delta=\left\{(v, v, \ldots, v) \mid v \in \mathbb{E}^{3}\right\}
$$


and $\Delta^{\perp}$ be the orthogonal complement of $\Delta$ in $\left(\mathbb{E}^{3}\right)^{k}$. Then, every point $x \in\left(\mathbb{E}^{3}\right)^{k}$ decomposes in a unique way as $x=x_{\Delta}+x_{\perp}$ with $x_{\Delta} \in \Delta$ and $x_{\perp} \in \Delta^{\perp}$. Note that if $x(t+1)=x(t)+\Delta t v(t)$ then $x(t+1)_{\perp}=x(t)_{\perp}+\Delta t v(t)_{\perp}$. Similarly, if $v(t+1)=-\left(\operatorname{Id}-\frac{L_{x}}{\left\|L_{x}\right\|}\right) v(t)$ then

$$
v(t+1)_{\perp}=-\left(\operatorname{Id}-\frac{L_{x}}{\left\|L_{x}\right\|}\right) v(t)_{\perp}
$$

since $L_{x}(\Delta)=0$ and $L_{x}\left(\Delta^{\perp}\right) \subseteq \Delta^{\perp}$. Finally, note that for all $x \in\left(\mathbb{E}^{3}\right)^{k}$ the matrices $A_{x}$ and $A_{x_{\perp}}$ are equal. It follows that the projections over $\Delta^{\perp}$ of the solutions of (4) are the solutions of the restriction of (4) to $\Delta^{\perp}$. A similar remark holds for (7).

These projections over $\Delta^{\perp}$ are of the essence since we are interested on the differences $x_{i}-x_{j}$ and $v_{i}-v_{j}$, for $i \neq j$, rather than on the $x_{i}$ or $v_{i}$ themselves.

We denote $\Gamma=\frac{1}{2} \sum_{i \neq j}\left\|x_{i}-x_{j}\right\|^{2}$ and $\Lambda=\frac{1}{2} \sum_{i \neq j}\left\|v_{i}-v_{j}\right\|^{2}$. To better deal with these functions consider $Q:\left(\mathbb{E}^{3}\right)^{k} \times\left(\mathbb{E}^{3}\right)^{k} \rightarrow \mathbb{R}$ defined by

$$
Q(u, v)=\frac{1}{2} \sum_{i, j=1}^{k}\left\langle u_{i}-u_{j}, v_{i}-v_{j}\right\rangle
$$

Then $Q$ is bilinear, symmetric, and, when restricted to $\Delta^{\perp} \times \Delta^{\perp}$, positive definite. It follows that it defines an inner product $\langle,\rangle_{Q}$ on $\left(\mathbb{E}^{3}\right)^{k} / \Delta \simeq \Delta^{\perp}$. Now note that $\Lambda=\|v\|_{Q}^{2}$ and $\Gamma=\|x\|_{Q}^{2}$ and that $\Gamma(x)=\Gamma\left(x_{\perp}\right)$ and $\Lambda(v)=\Lambda\left(v_{\perp}\right)$.

Let $\boldsymbol{\nu}, \overline{\boldsymbol{\nu}}>0$ be such that, restricted to $\Delta^{\perp}$,

$$
\boldsymbol{\nu}\|\|^{2} \leq\|\|_{Q}^{2} \leq \bar{\nu}\|\|^{2} .
$$

Note that $\boldsymbol{\nu}, \overline{\boldsymbol{\nu}}$ depend only on $k$. We now show bounds for them in terms of $k$.

Lemma 1 For all $k \geq 2, \boldsymbol{\nu}(k) \geq \frac{1}{3 k}$ and $\overline{\boldsymbol{\nu}}(k) \leq 2 k(k-1)$.

Proof. $\quad$ By definition, $\overline{\boldsymbol{\nu}} \leq \max _{\|x\|=1}\|x\|_{Q}^{2}$. Since $\|x\|=1,\left\|x_{i}\right\| \leq 1$ for $i=1, \ldots, k$ and therefore, $\left\|x_{i}-x_{j}\right\|^{2} \leq 4$ for all $i \neq j$. This implies

$$
\|x\|_{Q}^{2} \leq \frac{1}{2} k(k-1) 4=2 k(k-1) .
$$

Also by definition, $\frac{1}{\nu} \leq \max _{\|x\|_{Q}=1}\|x\|^{2}$. Let $x \in \Delta^{\perp}$ such that $\|x\|_{Q}=1$. We claim that, for all $i \leq k$ and $\ell \leq 3,\left|x_{i \ell}\right|<1$. Assume the contrary. Then there exists $i_{0}$ and $\ell$ such that $\left|x_{i_{0} \ell}\right| \geq 1$. Without loss of generality, $x_{i_{0} \ell} \geq 1$. Since $\sum x_{i}=0$, there exists $i_{1}$ such that $x_{i_{1} \ell}<0$. But then

$$
\|x\|_{Q}^{2}=\frac{1}{2} \sum_{i \neq j}\left\|x_{i}-x_{j}\right\|^{2} \geq\left\|x_{i_{0}}-x_{i_{1}}\right\|^{2} \geq\left(x_{i_{0} \ell}-x_{i_{1} \ell}\right)^{2}>1
$$


contradicting $\|x\|_{Q}^{2}=1$. So the claim is proved. Finally

$$
\|x\|^{2}=\sum_{i=1}^{k} \sum_{\ell=1}^{3} x_{i \ell}^{2} \leq 3 k
$$

which shows $\frac{1}{\nu} \leq 3 k$.

Remark 2 The condition "the velocities $v_{i}(t)$ tend to a common limit $\widehat{v} \in \mathbb{E}^{3 \text { " in }}$ Theorem 1 is equivalent to the condition " $v_{\perp}(t) \rightarrow 0$." Also, the condition "the vectors $x_{i}-x_{j}$ tend to a limit vector $\widehat{x_{i j}}$, for all $i, j \leq k$ " is equivalent to " $x_{\perp}(t)$ tend to a limit vector $\widehat{x}$ in $\Delta^{\perp}$." This suggests that we are actually interested on the solutions of the systems induced by (4) and (7), respectively, on the space $\Delta^{\perp} \times \Delta^{\perp}$. Since, as we mentioned, these induced systems have the same form as (4) and (7), we will keep referring to them but we will consider them on $\Delta^{\perp} \times \Delta^{\perp}$. Actually, we will consider positions in

$$
X:=\left(\mathbb{E}^{3}\right)^{k} / \Delta \simeq \Delta^{\perp}
$$

and velocities in

$$
V:=\left(\mathbb{E}^{3}\right)^{k} / \Delta \simeq \Delta^{\perp}
$$

\section{Convergence in continuous time}

Proposition 2 Let $A$ be a symmetric matrix, $L=D-A$ its Laplacian, $\phi$ its Fiedler number, and $\mu=\min _{i \neq j} a_{i j}$. Then $\phi \geq \boldsymbol{\nu}$. In particular, if $a_{i j}=\eta\left(\left\|x_{i}-x_{j}\right\|^{2}\right)$ then

$$
\phi \geq \boldsymbol{\nu} \eta\left(\Gamma_{x}\right)
$$

Proof. For all $v \in V$

$$
\|L v\|\|v\| \geq\langle L v, v\rangle=\sum_{i, j=1}^{k} a_{i j}\left\|v_{i}-v_{j}\right\|^{2} \geq \mu\|v\|_{Q}^{2} \geq \boldsymbol{\nu} \mu\|v\|^{2} .
$$

It follows that $\|L v\| \geq \boldsymbol{\nu} \mu\|v\|$ and thus the statement.

In the following we fix a solution $(x, v)$ of $(7)$. At a time $t \in \mathbb{R}_{+}, x(t)$ and $v(t)$ are elements in $X$ and $V$, respectively. In particular, $x(t)$ determines an adjacency matrix $A_{x(t)}$. For notational simplicity we will denote this matrix by $A_{t}$ and its Laplacian and Fiedler number by $L_{t}$ and $\phi_{t}$, respectively. Similarly, we will write $\Lambda(t)$ and $\Gamma(t)$ for the values of $\Lambda$ and $\Gamma$, respectively, at $(v(t), x(t))$. Finally, we will write $\Gamma_{0}$ for $\Gamma(0)$ and similarly for $\Lambda_{0}$.

Denote $\Phi_{t}=\min _{\tau \in[0, t]} \phi_{\tau}$. 
Proposition 3 For all $t \geq 0$

$$
\Lambda(t) \leq \Lambda_{0} e^{-2 t \Phi_{t}}
$$

Proof. Let $\tau \in[0, t]$. Then

$$
\begin{aligned}
\Lambda^{\prime}(\tau) & =\frac{d}{d \tau}\langle v(\tau), v(\tau)\rangle_{Q} \\
& =2\left\langle v^{\prime}(\tau), v(\tau)\right\rangle_{Q} \\
& =-2\left\langle L_{\tau} v(\tau), v(\tau)\right\rangle_{Q} \\
& \leq-2 \phi_{x(\tau)} \Lambda(\tau) .
\end{aligned}
$$

Here we have used that $L_{\tau}$ is symmetric positive definite on $V$. Using this inequality,

$$
\left.\ln (\Lambda(\tau))\right|_{0} ^{t}=\int_{0}^{t} \frac{\Lambda^{\prime}(\tau)}{\Lambda(\tau)} d \tau \leq \int_{0}^{t}-2 \phi_{\tau} d \tau=-2 t \Phi_{t}
$$

i.e.,

$$
\ln (\Lambda(t))-\ln \left(\Lambda_{0}\right) \leq-2 t \Phi_{t}
$$

from which the statement follows.

Proposition 4 For $T>0$

$$
\Gamma(T) \leq 2\left(\Gamma_{0}+\frac{\Lambda_{0}}{\Phi_{T}^{2}}\right)
$$

Proof. We have $\left|\Gamma^{\prime}(t)\right|=\left|2\langle v(t), x(t)\rangle_{Q}\right| \leq 2\|v(t)\|_{Q}\|x(t)\|_{Q}$. But $\|x(t)\|_{Q}=$ $\Gamma(t)^{1 / 2}$ and $\|v(t)\|_{Q}^{2}=\Lambda(t) \leq \Lambda_{0} e^{-2 t \Phi_{t}}$, by Proposition 3. Therefore,

$$
\Gamma^{\prime}(t) \leq\left|\Gamma^{\prime}(t)\right| \leq 2\left(\Lambda_{0} e^{-2 t \Phi_{t}}\right)^{1 / 2} \Gamma(t)^{1 / 2}
$$

and, using that $t \mapsto \Phi_{t}$ is non-increasing,

$$
\begin{aligned}
\int_{0}^{T} \frac{\Gamma^{\prime}(t)}{\Gamma(t)^{1 / 2}} d t & \leq 2 \int_{0}^{T}\left(\Lambda_{0} e^{-2 t \Phi_{t}}\right)^{1 / 2} d t \\
& \leq 2 \int_{0}^{T} \Lambda_{0}^{1 / 2} e^{-t \Phi_{T}} d t \\
& =\left.2 \Lambda_{0}^{1 / 2}\left(-\frac{1}{\Phi_{T}}\right) e^{-t \Psi(T)}\right|_{0} ^{T} \leq \frac{2 \Lambda_{0}^{1 / 2}}{\Phi_{T}}
\end{aligned}
$$

which implies

$$
\left.\Gamma(t)^{1 / 2}\right|_{0} ^{T}=\frac{1}{2} \int_{0}^{T} \frac{\Gamma^{\prime}(t)}{\Gamma(t)} d t \leq \frac{\Lambda_{0}^{1 / 2}}{\Phi_{T}}
$$


from which it follows that

$$
\Gamma(T) \leq\left(\Gamma_{0}^{1 / 2}+\frac{\Lambda_{0}^{1 / 2}}{\Phi_{T}}\right)^{2}
$$

The statement now follows from the elementary inequality $(\alpha+\beta)^{2} \leq 2\left(\alpha^{2}+\beta^{2}\right)$.

A proof of the following lemma is in [1, Lemma 7].

Lemma 2 Let $c_{1}, c_{2}>0$ and $s>q>0$. Then the equation

$$
F(z)=z^{s}-c_{1} z^{q}-c_{2}=0
$$

has a unique positive zero $z_{*}$. In addition

$$
z_{*} \leq \max \left\{\left(2 c_{1}\right)^{\frac{1}{s-q}},\left(2 c_{2}\right)^{\frac{1}{s}}\right\}
$$

and $F(z) \leq 0$ for $0 \leq z \leq z^{*}$.

Theorem 2 Assume that there are constants $K, \sigma>0$ and $\beta \geq 0$ and

$$
a_{i j}=\frac{K}{\left(\sigma^{2}+\left\|x_{i}-x_{j}\right\|^{2}\right)^{\beta}} .
$$

Assume also that one of the three following hypothesis hold:

(i) $\beta<1 / 2$,

(ii) $\beta=1 / 2$ and $\Lambda_{0}<\frac{(\boldsymbol{\nu} K)^{2}}{2}$,

(iii) $\beta>1 / 2$ and

$$
\left[\left(\frac{1}{2 \beta}\right)^{\frac{1}{2 \beta-1}}-\left(\frac{1}{2 \beta}\right)^{\frac{2 \beta}{2 \beta-1}}\right]\left(\frac{(\nu K)^{2}}{2 \Lambda_{0}}\right)^{\frac{1}{2 \beta-1}}>2 \Gamma_{0}+\sigma^{2} .
$$

Then there exists a constant $B_{0}$ (independent of $t$, made explicit in the proof of each of the three cases) such that $\Gamma(t) \leq B_{0}$ for all $t \in \mathbb{R}_{+}$. In addition, $\Lambda(t) \rightarrow 0$ when $t \rightarrow \infty$. Finally, there exists $\widehat{x} \in X$ such that $x(t) \rightarrow \widehat{x}$ when $t \rightarrow \infty$.

Proof. By Proposition 2, for all $x \in X$,

$$
\phi_{x} \geq \frac{\nu K}{\left(\sigma^{2}+\max _{i \neq j}\left\|x_{i}-x_{j}\right\|^{2}\right)^{\beta}} \geq \frac{\nu K}{\left(\sigma^{2}+\Gamma_{x}\right)^{\beta}} .
$$


Let $t^{*} \in[0, t]$ be the point maximizing $\Gamma$ in $[0, t]$. Then

$$
\Phi_{t}=\min _{\tau \in[0, t]} \phi_{\tau} \geq \min _{\tau \in[0, t]} \frac{\boldsymbol{\nu} K}{\left(\sigma^{2}+\Gamma(\tau)\right)^{\beta}} \geq \frac{\boldsymbol{\nu} K}{\left(\sigma^{2}+\Gamma\left(t^{*}\right)\right)^{\beta}} .
$$

By Proposition 4

$$
\Gamma(t) \leq 2 \Gamma_{0}+2 \Lambda_{0} \frac{\left(\sigma^{2}+\Gamma\left(t^{*}\right)\right)^{2 \beta}}{(\boldsymbol{\nu} K)^{2}} .
$$

Since $t^{*}$ maximizes $\Gamma$ in $[0, t]$ it also does so in $\left[0, t^{*}\right]$. Thus, for $t=t^{*},(9)$ takes the form

$$
\left(\sigma^{2}+\Gamma\left(t^{*}\right)\right)-2 \Lambda_{0} \frac{\left(\sigma^{2}+\Gamma\left(t^{*}\right)\right)^{2 \beta}}{(\boldsymbol{\nu} K)^{2}}-\left(2 \Gamma_{0}+\sigma^{2}\right) \leq 0 .
$$

Let $z=\Gamma\left(t^{*}\right)+\sigma^{2}$

$$
\boldsymbol{a}=\frac{2 \Lambda_{0}}{(\boldsymbol{\nu} K)^{2}}, \quad \text { and } \quad \boldsymbol{b}=2 \Gamma_{0}+\sigma^{2} .
$$

Then (10) can be rewritten as $F(z) \leq 0$ with

$$
F(z)=z-\boldsymbol{a} z^{2 \beta}-\boldsymbol{b} .
$$

(i) Assume $\beta<1 / 2$. By Lemma 2, $F(z) \leq 0$ implies that $z=\left(\sigma^{2}+\Gamma\left(t^{*}\right)\right) \leq U_{0}$ with

$$
U_{0}=\max \left\{\left(\frac{4 \Lambda_{0}}{(\boldsymbol{\nu} K)^{2}}\right)^{\frac{1}{1-2 \beta}}, 2\left(2 \Gamma_{0}+\sigma^{2}\right)\right\} .
$$

That is $\Gamma\left(t^{*}\right) \leq B_{0}:=U_{0}-\sigma^{2}$. Since $B_{0}$ is independent of $t$, we deduce that, for all $t \in \mathbb{R}_{+}, \Gamma(t) \leq B_{0}$. But this implies that $\phi_{t} \geq \frac{\boldsymbol{\nu} K}{\left(\sigma^{2}+B_{0}\right)^{\beta}}$ for all $t \in \mathbb{R}_{+}$and therefore, the same bound holds for $\Phi_{t}$. By Proposition 3

$$
\Lambda(t) \leq \Lambda_{0} e^{-2 \frac{\nu K}{\left(\sigma^{2}+B_{0}\right)^{\beta}} t}
$$

which shows that $\Lambda(t) \rightarrow 0$ when $t \rightarrow \infty$. Finally, for all $T>t$,

$$
\begin{aligned}
\|x(T)-x(t)\| & =\left\|\int_{t}^{T} v\right\| \leq \int_{t}^{T}\|v\| \leq \int_{t}^{T} \frac{1}{\nu} \Lambda^{1 / 2} \\
& \leq \int_{t}^{T} \frac{1}{\nu} \Lambda_{0}^{1 / 2} e^{-\frac{\nu K}{\left(\sigma^{2}+B_{0}\right)^{\beta}} s} d s=\left.\frac{1}{\nu} \Lambda_{0}^{1 / 2}\left(-\frac{\left(\sigma^{2}+B_{0}\right)^{\beta}}{\boldsymbol{\nu} K} e^{-\frac{\boldsymbol{\nu} K}{\left(\sigma^{2}+B_{0}\right)^{\beta}} s}\right)\right|_{t} ^{T} \\
& =\frac{\Lambda_{0}^{1 / 2}\left(\sigma^{2}+B_{0}\right)^{\beta}}{\boldsymbol{\nu}^{2} K}\left(e^{-\frac{\nu K}{\left(\sigma^{2}+B_{0}\right)^{\beta}} t}-e^{-\frac{\nu K}{\left(\sigma^{2}+B_{0}\right)^{\beta}} T}\right) \\
& \leq \frac{\Lambda_{0}^{1 / 2}\left(\sigma^{2}+B_{0}\right)^{\beta}}{\boldsymbol{\nu}^{2} K} e^{-\frac{\nu K}{\left(\sigma^{2}+B_{0}\right)^{\beta}} t} .
\end{aligned}
$$


Since the last tend to zero with $t$ and is independent of $T$ we deduce that there exists $\widehat{x} \in X$ such that, $x \rightarrow \widehat{x}$.

(ii) Assume now $\beta=1 / 2$. Then (10) takes the form

$$
\left(\sigma^{2}+\Gamma\left(t^{*}\right)\right)\left(1-\frac{2 \Lambda_{0}}{(\boldsymbol{\nu} K)^{2}}\right)-\left(2 \Gamma_{0}+\sigma^{2}\right) \leq 0
$$

which implies that

$$
\Gamma\left(t^{*}\right) \leq B_{0}:=\frac{2 \Gamma_{0}+\sigma^{2}}{1-\frac{2 \Lambda_{0}}{(\boldsymbol{\nu} K)^{2}}}-\sigma^{2} .
$$

Note that $B_{0}>0$ since $\Lambda_{0}<\frac{(\boldsymbol{\nu} K)^{2}}{2}$. We now proceed as in case (i).

(iii) Assume finally $\beta>1 / 2$ and let $\alpha=2 \beta$ so that $F(z)=z-\boldsymbol{a} z^{\alpha}-\boldsymbol{b}$. The derivative $F^{\prime}(z)=1-\alpha \boldsymbol{a} z^{\alpha-1}$ has a unique zero at $z_{*}=\left(\frac{1}{\alpha \boldsymbol{a}}\right)^{\frac{1}{\alpha-1}}$ and

$$
\begin{aligned}
F\left(z_{*}\right) & =\left(\frac{1}{\alpha \boldsymbol{a}}\right)^{\frac{1}{\alpha-1}}-\boldsymbol{a}\left(\frac{1}{\alpha \boldsymbol{a}}\right)^{\frac{\alpha}{\alpha-1}}-\boldsymbol{b} \\
& =\left(\frac{1}{\alpha}\right)^{\frac{1}{\alpha-1}}\left(\frac{1}{\boldsymbol{a}}\right)^{\frac{1}{\alpha-1}}-\left(\frac{1}{\alpha}\right)^{\frac{\alpha}{\alpha-1}}\left(\frac{1}{\boldsymbol{a}}\right)^{\frac{1}{\alpha-1}}-\boldsymbol{b} \\
& =\left(\frac{1}{\boldsymbol{a}}\right)^{\frac{1}{\alpha-1}}\left[\left(\frac{1}{\alpha}\right)^{\frac{1}{\alpha-1}}-\left(\frac{1}{\alpha}\right)^{\frac{\alpha}{\alpha-1}}\right]-\boldsymbol{b} \\
& \geq 0
\end{aligned}
$$

the last by our hypothesis. Since $F(0)=-\boldsymbol{b}<0$ and $F(z) \rightarrow-\infty$ when $z \rightarrow \infty$ we deduce that the shape of $F$ is as follows:

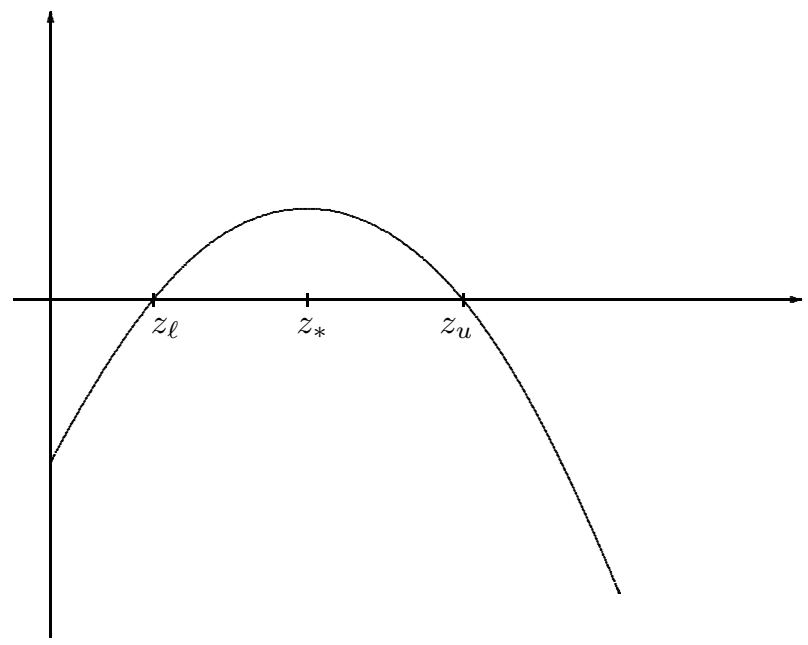

Figure 1 
Even though $t^{*}$ is not continuous as a function of $t$, the mapping $t \mapsto \Gamma\left(t^{*}\right)+\sigma^{2}$ is continuous and therefore, so is the mapping $t \mapsto F\left(\Gamma\left(t^{*}\right)+\sigma^{2}\right)$. This fact, together with (10), shows that, for all $t \geq 0, F\left(\Gamma\left(t^{*}\right)+\sigma^{2}\right) \leq 0$. In addition, when $t=0$ we have $t^{*}=0$ as well and

$$
\begin{aligned}
\Gamma_{0}+\sigma^{2} & \leq 2 \Gamma_{0}+\sigma^{2}=\boldsymbol{b} \\
& <\left(\frac{1}{\boldsymbol{a}}\right)^{\frac{1}{\alpha-1}}\left[\left(\frac{1}{\alpha}\right)^{\frac{1}{\alpha-1}}-\left(\frac{1}{\alpha}\right)^{\frac{\alpha}{\alpha-1}}\right] \\
& <\left(\frac{1}{\boldsymbol{a}}\right)^{\frac{1}{\alpha-1}}\left(\frac{1}{\alpha}\right)^{\frac{1}{\alpha-1}} \\
& =z_{*} .
\end{aligned}
$$

This implies that $\Gamma_{0}+\sigma^{2}<z_{\ell}$ (the latter being the smallest zero of $F$ on $\mathbb{R}_{+}$, see the figure above) and the continuity of the map $t \mapsto \Gamma\left(t^{*}\right)+\sigma^{2}$ implies that, for all $t \geq 0$

$$
\Gamma\left(t^{*}\right)+\sigma^{2} \leq z_{\ell} \leq z_{*}
$$

Therefore

$$
\Gamma\left(t^{*}\right) \leq B_{0}:=\left(\frac{1}{\alpha \boldsymbol{a}}\right)^{\frac{1}{\alpha-1}}-\sigma^{2}=\left(\frac{(\boldsymbol{\nu} K)^{2}}{2 \alpha \Lambda_{0}}\right)^{\frac{1}{\alpha-1}}-\sigma^{2} .
$$

We now proceed as in case (i).

Remark 3 (i) In Theorem 2, the condition that $a_{i j}=\frac{K}{\left(\sigma^{2}+\left\|x_{i}-x_{j}\right\|^{2}\right)^{\beta}}$ may be relaxed to $a_{i j} \geq \frac{K}{\left(\sigma^{2}+\left\|x_{i}-x_{j}\right\|^{2}\right)^{\beta}}$.

(ii) The bound $\beta<1 / 2$ for unconditional convergence in Theorem 2 is essentially sharp. We will indicate this in Remark 4 below by studying the special case of a flock with two birds flying on a line.

\section{A flock of two birds}

We give here a more detailed analysis of the case of two birds flying on a line (i.e., we take $\mathbb{R}$ instead of $\mathbb{E}^{3}$ for both positions and velocities).

We define $\boldsymbol{x}=x_{1}-x_{2}$ and $\boldsymbol{v}=v_{1}-v_{2}$ and assume that the state $(\boldsymbol{x}, \boldsymbol{v})$ of the pair satisfies the system of ODE's

$$
\begin{aligned}
\boldsymbol{x}^{\prime} & =\boldsymbol{v} \\
\boldsymbol{v}^{\prime} & =-\frac{\boldsymbol{v}}{(1+\boldsymbol{x})^{\alpha}} .
\end{aligned}
$$

This is not exactly (7) but it is easier to dealt with and, we will see below, it is close to this system. 
The arguments used in the preceding section show that when $\alpha<1$, for all initial $\boldsymbol{x}_{0}$ and $\boldsymbol{v}_{0}$, we have that $\boldsymbol{x}$ is bounded and $\boldsymbol{v} \rightarrow 0$ when $t \rightarrow \infty$. The next proposition gives conditions on $\boldsymbol{x}_{0}$ and $\boldsymbol{v}_{0}$ for such a convergence to hold when $\alpha>1$.

Proposition 5 Let $\alpha>1$. Assume that $\boldsymbol{x}_{0}>0$ and $\boldsymbol{v}_{0}>0$ and that

$$
\boldsymbol{x}_{0}<\widehat{\boldsymbol{x}_{0}}:=\left(\frac{\alpha-1}{\boldsymbol{v}_{0}}\right)^{\frac{1}{\alpha-1}}+1
$$

Then $\boldsymbol{x}$ is bounded and increasing. In addition, when $t \rightarrow \infty, \boldsymbol{v}(t) \rightarrow 0$ and

$$
\boldsymbol{x}(t) \rightarrow\left(\frac{\alpha-1}{\boldsymbol{v}_{0}-\frac{\alpha-1}{\left(1+\boldsymbol{x}_{0}\right)^{\alpha-1}}}\right)^{\frac{1}{\alpha-1}}+1
$$

Proof. It follows from the system (12) that, for all $t \geq 0$,

$$
\int_{0}^{t} \boldsymbol{v}^{\prime}=\int_{0}^{t} \frac{\boldsymbol{x}^{\prime}}{(1+\boldsymbol{x})^{\alpha}}
$$

and therefore, integrating both sides between 0 and $t$, that

$$
\boldsymbol{v}(t)-\boldsymbol{v}_{0}=\frac{\alpha-1}{(1+\boldsymbol{x}(t))^{\alpha-1}}-\frac{\alpha-1}{\left(1+\boldsymbol{x}_{0}\right)^{\alpha-1}}
$$

or yet, that

$$
\boldsymbol{v}(t)=\frac{\alpha-1}{(1+\boldsymbol{x}(t))^{\alpha-1}}-\gamma_{0}
$$

where $\gamma_{0}>0$ since $\boldsymbol{x}_{0}<\widehat{\boldsymbol{x}_{0}}$.

If, for some $t_{*}, \boldsymbol{v}\left(t_{*}\right)=0$ then $\boldsymbol{v}^{\prime}\left(t_{*}\right)=0$. But then the pair $(\widetilde{x}, \widetilde{v})$ defined by $\widetilde{x}_{1}(t)=x_{1}\left(t_{*}\right), \widetilde{x}_{2}(t)=x_{2}\left(t_{*}\right)$ and $\widetilde{v}(t)=0$, for all $t \geq 0$, is a solution of (12) satisfying the conditions $\widetilde{\boldsymbol{x}}\left(t_{*}\right)=\boldsymbol{x}\left(t_{*}\right)$ and $\widetilde{\boldsymbol{v}}=0$. By the unicity of the solutions of (12) it follows that $\widetilde{\boldsymbol{v}}=\boldsymbol{v}$ and hence that $\widetilde{\boldsymbol{v}}_{0}=0$ in contradiction with our assumptions. We conclude that $\boldsymbol{v}(t)>0$ for all $t \geq 0$. But then

$$
0<\boldsymbol{v}(t)=\frac{\alpha-1}{(1+\boldsymbol{x}(t))^{\alpha-1}}-\gamma_{0}
$$

implies that

$$
\boldsymbol{x}(t)<\left(\frac{\alpha-1}{\gamma_{0}}\right)^{\frac{1}{\alpha-1}}+1 .
$$

Thus, $\boldsymbol{x}$ remains bounded on $\mathbb{R}_{+}$. Furthermore $\boldsymbol{x}$ is increasing since $\boldsymbol{v}>0$. This implies that there exists $\boldsymbol{x}_{*}>0$ such that $\boldsymbol{x}(t) \rightarrow \boldsymbol{x}_{*}$ and $\boldsymbol{x}^{\prime}(t) \rightarrow 0$ when $t \rightarrow \infty$. It follows from $\boldsymbol{x}^{\prime}=\boldsymbol{v}$ and (13) that $\boldsymbol{x}_{*}$ is as claimed. 
Remark 4 It follows from the proof of Proposition 5 that, for all $\alpha>1, \boldsymbol{v}$ fails to converge if $\boldsymbol{x} \geq \widehat{\boldsymbol{x}_{0}}$. Also, since

$$
\frac{1}{(1+\boldsymbol{x})^{\alpha}} \leq \frac{1}{\left(1+\boldsymbol{x}^{2}\right)^{\beta}} \leq 2 \frac{\sqrt{2}}{(1+\boldsymbol{x})^{\alpha}}
$$

the system (7) is tightly bounded in between two versions of (12) differing only by a constant factor. This indicates that convergence may fail as well in (7) for $\alpha>1$.

\section{Convergence in discrete time}

We now focus on discrete time. The model is thus (4). A motivation to consider discrete time is that we want to derive (possibly a small variation of) our model from a mechanism based on exchanges of signals. The techniques to do so, learning theory, are better adapted to discrete time. Also, we want our model to include noisy environments and this issue becomes more technically involved in continuous time.

We assume as before that there are constants $K, \sigma>0$ and $\beta \geq 0$ such that

$$
a_{i j}=\frac{K}{\left(\sigma^{2}+\left\|x_{i}-x_{j}\right\|^{2}\right)^{\beta}} .
$$

Note that, by Proposition 2, this implies that $\phi_{x}>0$ for all $x \in X$. This, in turn, shows that $L_{x}$ is a self-adjoint, positive definite linear map, whose smallest eigenvalue is $\phi_{x}$. We denote by $\kappa(x)$ its condition number, i.e.,

$$
\kappa(x)=\frac{\left\|L_{x}\right\|}{\phi_{x}} .
$$

Lemma 3 For all $x \in X$,

$$
\kappa(x) \leq \frac{k\left(\sigma^{2}+\Gamma_{x}\right)^{\beta}}{\nu \sigma^{2 \beta}}
$$

Proof. $\quad$ By Proposition 2

$$
\phi_{x} \geq \boldsymbol{\nu} \min _{i, j} a_{i j} \geq \boldsymbol{\nu} \frac{K}{\left(\sigma^{2}+\Gamma_{x}\right)^{\beta}} .
$$

Also, $\left\|L_{x}\right\| \leq \frac{K k}{\sigma^{2 \beta}}$ since all of its entries are bounded by $\frac{K}{\sigma^{2 \beta}}$.

In the following we fix a solution $(x, v)$ of (4). At a time $t \in \mathbb{N}, x(t)$ and $v(t)$ are elements in $X$ and $V$, respectively. The meaning of expressions like $\phi_{t}, L_{t}, \kappa(t)$, or $\Gamma(t)$ is as described in Section 3 .

Proposition 6 For all $t \in \mathbb{N},\|v(t+1)\| \leq\left(1-\frac{1}{\kappa(t)}\right)\|v(t)\|$. In particular, $\|v\|$ is decreasing as a function of $t$. 
Proof. The linear map Id $-\frac{1}{\left\|L_{t}\right\|} L_{t}$ is self-adjoint and its eigenvalues are in the interval $[0,1)$. In addition, its largest eigenvalue is $1-\frac{\phi_{t}}{\left\|L_{t}\right\|}$. Therefore

$$
\|v(t+1)\|=\left\|\left(\mathrm{Id}-\frac{1}{\left\|L_{t}\right\|} L_{t}\right) v(t)\right\| \leq\left(1-\frac{\phi_{t}}{\left\|L_{t}\right\|}\right)\|v(t)\|
$$

Corollary 1 For all $t \in \mathbb{N},\|v(t)\| \leq \prod_{i=0}^{t-1}\left(1-\frac{1}{\kappa(i)}\right)\|v(0)\|$.

Theorem 3 Assume that there are constants $K, \sigma>0$ and $\beta \geq 0$ such that

$$
a_{i j}=\frac{K}{\left(\sigma^{2}+\left\|x_{i}-x_{j}\right\|^{2}\right)^{\beta}} .
$$

Assume also that one of the three following hypothesis hold:

(i) $\beta<1 / 2$,

(ii) $\beta=1 / 2$ and $\|v(0)\| \leq \frac{\boldsymbol{\nu} \sigma^{2 \beta}}{k \overline{\boldsymbol{\nu}}^{1 / 2} \Delta t}$,

(iii) $\beta>1 / 2$ and

$$
\left(\frac{1}{\boldsymbol{a}}\right)^{\frac{2}{\alpha-1}}\left[\left(\frac{1}{\alpha}\right)^{\frac{2}{\alpha-1}}-\left(\frac{1}{\alpha}\right)^{\frac{\alpha+1}{\alpha-1}}\right]>\overline{\boldsymbol{\nu}}\left(V_{0}^{2}+2 V_{0}\left((\alpha \boldsymbol{a})^{-\frac{2}{\alpha-1}}-\sigma^{2}\right) \overline{\boldsymbol{\nu}}^{-1 / 2}\right)+\boldsymbol{b}
$$

Here $\alpha=2 \beta, V_{0}:=\Delta t\|v(0)\|$,

$$
\boldsymbol{a}=\frac{k \overline{\boldsymbol{\nu}}^{1 / 2}}{\boldsymbol{\nu} \sigma^{2 \beta}} V_{0}, \quad \text { and } \quad \boldsymbol{b}=\overline{\boldsymbol{\nu}}^{1 / 2}\|x(0)\|+\sigma .
$$

Then there exists a constant $B_{0}$ (independent of $t$, made explicit in the proof of each of the three cases) such that $\|x(t)\| \leq B_{0}$ for all $t \in \mathbb{N}$. In addition, $\|v(t)\| \rightarrow 0$ when $t \rightarrow \infty$. Finally, there exists $\widehat{x} \in X$ such that $x(t) \rightarrow \widehat{x}$ when $t \rightarrow \infty$.

Proof. For $t \in \mathbb{N}$ let $t^{*}$ be the point maximizing $\|x\|$ in $\{0,1, \ldots, t\}$. Then, by Lemma 3 , for $i \in\{0,1, \ldots, t\}$,

$$
\kappa(i) \leq \frac{k\left(\sigma^{2}+\Gamma(i)\right)^{\beta}}{\boldsymbol{\nu} \sigma^{2 \beta}} \leq \frac{k\left(\sigma^{2}+\overline{\boldsymbol{\nu}}\|x(i)\|^{2}\right)^{\beta}}{\boldsymbol{\nu} \sigma^{2 \beta}} \leq H\left(t^{*}\right):=\frac{k\left(\sigma^{2}+\overline{\boldsymbol{\nu}}\left\|x\left(t^{*}\right)\right\|^{2}\right)^{\beta}}{\boldsymbol{\nu} \sigma^{2 \beta}} .
$$


Using Corollary 1 we obtain

$$
\begin{aligned}
\|x(t)\| & \leq\|x(0)\|+\sum_{j=0}^{t-1}\|x(j+1)-x(j)\| \\
& \leq\|x(0)\|+\Delta t \sum_{j=0}^{t-1}\|v(j)\| \\
& \leq\|x(0)\|+\Delta t\left(\|v(0)\|+\sum_{j=1}^{t-1}\|v(j)\|\right) \\
& \leq\|x(0)\|+\Delta t\left(\|v(0)\|+\sum_{j=1}^{t-1} \prod_{i=1}^{j}\left(1-\frac{1}{\kappa(i)}\right)\|v(0)\|\right) \\
& \leq\|x(0)\|+\Delta t \sum_{j=0}^{t-1}\left(1-\frac{1}{H\left(t^{*}\right)}\right)^{j}\|v(0)\| \\
& \leq\|x(0)\|+\Delta t H\left(t^{*}\right)\|v(0)\| \\
& =\|x(0)\|+\Delta t \frac{k\left(\sigma^{2}+\bar{\nu}\left\|x\left(t^{*}\right)\right\|^{2}\right)^{\beta}}{\boldsymbol{\nu} \sigma^{2 \beta}}\|v(0)\| .
\end{aligned}
$$

Since $t^{*}$ maximizes $\|x\|$ in $\{0,1, \ldots, t\}$ it also does so in $\left\{0,1, \ldots, t^{*}\right\}$. For $t=t^{*}$, the inequality above takes then the following equivalent form

$$
\sigma+\overline{\boldsymbol{\nu}}^{1 / 2}\left\|x\left(t^{*}\right)\right\| \leq\left(\overline{\boldsymbol{\nu}}^{1 / 2}\|x(0)\|+\sigma\right)+\overline{\boldsymbol{\nu}}^{1 / 2} \Delta t \frac{k\left(\sigma^{2}+\overline{\boldsymbol{\nu}}\left\|x\left(t^{*}\right)\right\|^{2}\right)^{\beta}}{\boldsymbol{\nu} \sigma^{2 \beta}}\|v(0)\|
$$

which implies

$$
\left(\sigma^{2}+\overline{\boldsymbol{\nu}}\left\|x\left(t^{*}\right)\right\|^{2}\right)^{1 / 2} \leq\left(\overline{\boldsymbol{\nu}}^{1 / 2}\|x(0)\|+\sigma\right)+\overline{\boldsymbol{\nu}}^{1 / 2} \Delta t \frac{k\left(\sigma^{2}+\overline{\boldsymbol{\nu}}\left\|x\left(t^{*}\right)\right\|^{2}\right)^{\beta}}{\boldsymbol{\nu} \sigma^{2 \beta}}\|v(0)\| .
$$

Let $z=\left(\sigma^{2}+\bar{\nu}\left\|x\left(t^{*}\right)\right\|^{2}\right)^{1 / 2}$,

$$
\boldsymbol{a}=\frac{k \overline{\boldsymbol{\nu}}^{1 / 2} \Delta t}{\boldsymbol{\nu} \sigma^{2 \beta}}\|v(0)\|, \quad \text { and } \quad \boldsymbol{b}=\overline{\boldsymbol{\nu}}^{1 / 2}\|x(0)\|+\sigma .
$$

Then (14) can be rewritten as $F(z) \leq 0$ with

$$
F(z)=z-\boldsymbol{a} z^{2 \beta}-\boldsymbol{b} .
$$

(i) Assume $\beta<1 / 2$. By Lemma $2, F(z) \leq 0$ implies that $\left(\sigma^{2}+\bar{\nu}\left\|x\left(t^{*}\right)\right\|^{2}\right) \leq U_{0}^{2}$ with

$$
U_{0}=\max \left\{\left(\frac{2 k \overline{\boldsymbol{\nu}}^{1 / 2} \Delta t}{\boldsymbol{\nu} \sigma^{2 \beta}}\|v(0)\|\right)^{\frac{1}{1-2 \beta}}, 2\left(\overline{\boldsymbol{\nu}}^{1 / 2}\|x(0)\|+\sigma\right)\right\} .
$$


Since $U_{0}$ is independent of $t$ we deduce that, for all $t \in \mathbb{N}$,

$$
\|x(t)\|^{2} \leq B_{0}^{2}:=\frac{U_{0}^{2}-\sigma^{2}}{\bar{\nu}}
$$

and therefore, by Lemma 3,

$$
\kappa(t) \leq \frac{k\left(\sigma^{2}+\Gamma(t)\right)^{\beta}}{\boldsymbol{\nu} \sigma^{2 \beta}} \leq \frac{k\left(\sigma^{2}+\overline{\boldsymbol{\nu}}\|x(t)\|^{2}\right)^{\beta}}{\boldsymbol{\nu} \sigma^{2 \beta}} \leq \kappa_{*}:=\frac{k U_{0}^{2 \beta}}{\boldsymbol{\nu} \sigma^{2 \beta}} .
$$

By Corollary 1 , for $t \in \mathbb{N}$,

$$
\|v(t)\| \leq \prod_{i=0}^{t-1}\left(1-\frac{1}{\kappa(i)}\right)\|v(0)\| \leq\left(1-\frac{1}{\kappa_{*}}\right)^{t}\|v(0)\|
$$

and this expression tends to zero when $t \rightarrow \infty$.

Finally, for $T>t$, reasoning as above, we have

$$
\begin{aligned}
\|x(T)-x(t)\| & \leq \sum_{j=t}^{T-1}\|x(j+1)-x(j)\| \leq \Delta t \sum_{j=t}^{T-1}\|v(j)\| \\
& \leq \Delta t \sum_{j=t}^{T-1}\left(1-\frac{1}{\kappa_{*}}\right)^{j}\|v(t)\| \leq \Delta t \kappa_{*}\|v(t)\| .
\end{aligned}
$$

Since $\|v(t)\|$ tends to zero, we deduce that $\{x(t)\}_{t \in \mathbb{N}}$ is a Cauchy sequence and there exists $\widehat{x} \in X$ such that $x(t) \rightarrow \widehat{x}$.

(ii) Assume now $\beta=1 / 2$. Then (14) takes the form

$$
\left(\sigma^{2}+\overline{\boldsymbol{\nu}}\left\|x\left(t^{*}\right)\right\|^{2}\right)^{1 / 2}\left(1-\frac{k \overline{\boldsymbol{\nu}}^{1 / 2} \Delta t}{\boldsymbol{\nu} \sigma^{2 \beta}}\|v(0)\|\right)-\left(\overline{\boldsymbol{\nu}}^{1 / 2}\|x(0)\|+\sigma\right) \leq 0
$$

which implies that

$$
\left\|x\left(t^{*}\right)\right\|^{2} \leq B_{0}:=\frac{1}{\overline{\boldsymbol{\nu}}}\left(\left(\frac{\overline{\boldsymbol{\nu}}^{1 / 2}\|x(0)\|+\sigma}{1-\frac{k \overline{\boldsymbol{\nu}}^{1 / 2} \Delta t}{\boldsymbol{\nu} \sigma^{2 \beta}}\|v(0)\|}\right)^{2}-\sigma^{2}\right)
$$

which is positive since, by hypothesis, $0<1-\frac{k \overline{\boldsymbol{\nu}}^{1 / 2} \Delta t}{\boldsymbol{\nu} \sigma^{2 \beta}}\|v(0)\| \leq 1$. We now proceed as in case (i).

(iii) Assume finally $\beta>1 / 2$. Letting $\alpha=2 \beta$ as in the proof of Theorem 2 , the arguments therein show that the derivative $F^{\prime}(z)=1-\alpha \boldsymbol{a} z^{\alpha-1}$ has a unique zero at $z_{*}=\left(\frac{1}{\alpha \boldsymbol{a}}\right)^{\frac{1}{\alpha-1}}$ and $F\left(z_{*}\right)=\left(\frac{1}{\boldsymbol{a}}\right)^{\frac{1}{\alpha-1}}\left[\left(\frac{1}{\alpha}\right)^{\frac{1}{\alpha-1}}-\left(\frac{1}{\alpha}\right)^{\frac{\alpha}{\alpha-1}}\right]-\boldsymbol{b}$. Our hypothesis then implies that $F\left(z_{*}\right) \geq 0$. This shows that the graph of $F$ is as in Figure 1. 
For $t \in \mathbb{N}$ let $z(t)=\left(\sigma^{2}+\overline{\boldsymbol{\nu}}\left\|x\left(t^{*}\right)\right\|^{2}\right)^{1 / 2}$. When $t=0$ we have $t^{*}=0$ as well and

$$
z(0) \leq \overline{\boldsymbol{\nu}}^{1 / 2}\|x(0)\|+\sigma=\boldsymbol{b}<\left(\frac{1}{\boldsymbol{a}}\right)^{\frac{1}{\alpha-1}}\left(\frac{1}{\alpha}\right)^{\frac{1}{\alpha-1}}=z_{*} .
$$

This actually implies that $z(0) \leq z_{\ell}$. Assume that there exists $t \in \mathbb{N}$ such that $z(t) \geq z_{u}$ and let $T$ be the first such $t$. Then $T=T^{*} \geq 1$ and, for all $t<T$

$$
\left(\sigma^{2}+\bar{\nu}\|x(t)\|^{2}\right)^{1 / 2} \leq z(T-1) \leq z_{\ell} .
$$

This shows that, for all $t<T$,

$$
\|x(t)\| \leq\left(\frac{z_{\ell}^{2}-\sigma^{2}}{\overline{\boldsymbol{\nu}}}\right)^{1 / 2} \leq B_{0}:=\left(\frac{z_{*}^{2}-\sigma^{2}}{\overline{\boldsymbol{\nu}}}\right)^{1 / 2} .
$$

In particular,

$$
\|x(T-1)\|^{2} \leq \frac{z_{\ell}^{2}-\sigma^{2}}{\bar{\nu}}
$$

For $T$ instead, we have

$$
\|x(T)\|^{2} \geq \frac{z_{u}^{2}-\sigma^{2}}{\bar{\nu}}
$$

This implies

$$
\|x(T)\|^{2}-\|x(T-1)\|^{2} \geq \frac{z_{u}^{2}-z_{\ell}^{2}}{\overline{\boldsymbol{\nu}}} \geq \frac{z_{*}^{2}-z_{\ell}^{2}}{\overline{\boldsymbol{\nu}}} \geq \frac{\left(z_{*}-z_{\ell}\right) z_{*}}{\overline{\boldsymbol{\nu}}} .
$$

From the intermediate value theorem, there is $\xi \in\left[z_{\ell}, z_{*}\right]$ such that $F\left(z_{*}\right)=$ $F^{\prime}(\xi)\left(z_{*}-z_{\ell}\right)$. But $F^{\prime}(\xi) \geq 0$ and $F^{\prime}(\xi)=1-a \alpha \xi^{\alpha-1} \leq 1$. Therefore,

$$
z_{*}-z_{\ell} \geq F\left(z_{*}\right)
$$

and it follows from (15) that

$$
\|x(T)\|^{2}-\|x(T-1)\|^{2} \geq \frac{z_{*} F\left(z_{*}\right)}{\overline{\boldsymbol{\nu}}} .
$$

But

$$
\begin{aligned}
\|x(T)\|-\|x(T-1)\| & \leq\|x(T)-x(T-1)\| \\
& =\Delta t\|v(T-1)\| \\
& \leq \Delta t\|v(0)\|
\end{aligned}
$$

the last since $\|v\|$ is decreasing. Therefore,

$$
\begin{aligned}
\|x(T)\|^{2}-\|x(T-1)\|^{2} & \leq(\Delta t)^{2}\|v(0)\|^{2}+2 \Delta t\|v(0)\|\|x(T-1)\| \\
& \leq(\Delta t)^{2}\|v(0)\|^{2}+2 \Delta t\|v(0)\| B_{0} .
\end{aligned}
$$


Putting this inequality together with (16) shows that

$$
z_{*} F\left(z_{*}\right) \leq \overline{\boldsymbol{\nu}}\left((\Delta t)^{2}\|v(0)\|^{2}+2 \Delta t\|v(0)\| B_{0}\right)
$$

or equivalently,

$$
\left(\frac{1}{\boldsymbol{a}}\right)^{\frac{2}{\alpha-1}}\left[\left(\frac{1}{\alpha}\right)^{\frac{2}{\alpha-1}}-\left(\frac{1}{\alpha}\right)^{\frac{\alpha+1}{\alpha-1}}\right]-\boldsymbol{b} \leq \bar{\nu}\left((\Delta t)^{2}\|v(0)\|^{2}+2 \Delta t\|v(0)\| B_{0}\right)
$$

which contradicts our hypothesis.

We conclude that, for all $t \in \mathbb{N}, z(t) \leq z_{\ell}$ and hence, $\|x(t)\| \leq B_{0}$. We now proceed as in case (i).

Remark 5 (i) In the system (4) we could have replaced $\left\|L_{x}\right\|$ by $\frac{K k}{\sigma^{2 \beta}}$ and Theorem 3 would hold as well (with the same $B_{0}$ proved therein). Interpreting the latter choice would require less computational capabilities on the birds.

(ii) In the proof of Theorem 3 we could have used the bounds for $\bar{\nu}$ and $\boldsymbol{\nu}$ exhibited in Lemma 1 and, in case (iii) the trivial bound $(\alpha \boldsymbol{a})^{-\frac{2}{\alpha-1}}-\sigma^{2} \leq(\alpha \boldsymbol{a})^{-\frac{2}{\alpha-1}}$. Recall, $V_{0}:=\Delta t\|v(0)\|$. Denoting as well $X_{0}:=\|x(0)\|$ and

$$
g(\alpha):=\left[\left(\frac{1}{\alpha}\right)^{\frac{2}{\alpha-1}}-\left(\frac{1}{\alpha}\right)^{\frac{\alpha+1}{\alpha-1}}\right]
$$

the sufficiency condition for convergence in case (iii) becomes

$$
g(\alpha)\left(\frac{\sigma^{\alpha}}{3 \sqrt{2} k^{3}}\right)^{\frac{1}{\alpha-1}} V_{0}^{-\frac{1}{\alpha-1}} \geq \sqrt{2} k X_{0}+\sigma+2\left(k^{2} V_{0}^{2}+V_{0}^{\frac{\alpha-2}{\alpha-1}}\left(\frac{\sigma^{\alpha}}{3 \sqrt{2} k^{3}}\right)^{\frac{1}{\alpha-1}}\right) .
$$

It is apparent from the expression above that this condition is satisfied when $V_{0}$ is sufficiently small. It is also apparent that the larger $k$ is, the smaller $V_{0}$ needs to be to satisfy the condition.

We note also that, for $\alpha>1$, we have $0<g(\alpha)<1$ and that $g(a) \rightarrow 0$ when $\alpha \rightarrow 1$.

\section{Language evolution}

We now consider a linguistic population with $k$ agents evolving with time. At time $t$, the state of the population is given by $(x(t), f(t)) \in\left(\mathbb{E}^{3}\right)^{k} \times \mathcal{H}^{k}$. Here $\mathbb{E}^{3}$ is interpreted as the space of positions and $\mathcal{H}$ as the space of languages of [2]. Thus, unlike the development in Section 3, the functions $x$ and $f$ do not belong to the same space. 
We model the evolution of the population with the system of differential equations

$$
\begin{aligned}
x^{\prime} & =-L_{f} x \\
f^{\prime} & =-L_{x} f .
\end{aligned}
$$

Again, $L_{x}$ is the Laplacian of the matrix $A_{x}$ given by $a_{i j}=\eta_{X}\left(\left\|x_{i}-x_{j}\right\|^{2}\right)$ for some function $\eta_{X}: \mathbb{R}_{+} \rightarrow \mathbb{R}_{+}$. Similarly with $L_{f}$ for some function $\eta_{\mathcal{H}}: \mathbb{R}_{+} \rightarrow \mathbb{R}_{+}$. The distance between languages in $\mathcal{H}$ is defined as in [2].

A rationale for this model could be the following. Agents tend to move towards other agents using languages close to theirs (and therefore, communicating better). Hence, the first equation. Also, languages evolve by the influence from other agents' languages and this influence decrease with distance (for instance, because of a decrease in the frequency of linguistic encounters). Hence, the second equation.

Theorem 4 Let $\eta_{X}, \eta_{\mathcal{H}}: \mathbb{R}_{+} \rightarrow(0, \infty)$ be non-increasing. Then, when $t \rightarrow \infty$, the state $(x, f)$ tends to a point in the diagonal of $\left(\mathbb{E}^{3} \times \mathcal{H}\right)^{k}$.

Proof. We use the ideas and notations from Section 3. Reasoning as in Proposition 3 we obtain, for all $t>0$,

$$
\Lambda^{\prime}(f(t)) \leq-2 \phi_{t} \Lambda(f(t)) \quad \text { and } \quad \Gamma^{\prime}(x(t)) \leq-2 \phi_{f(t)} \Gamma(x(t)) .
$$

This shows that both $\Lambda$ and $\Gamma$ are decreasing and satisfy

$$
\Lambda(f(t)) \leq \Lambda_{0} e^{-2 \int_{0}^{t} \phi_{\tau} d \tau} \quad \text { and } \quad \Gamma(f(t)) \leq \Gamma_{0} e^{-2 \int_{0}^{t} \phi_{f(\tau)} d \tau} .
$$

But since both $\eta$ and $\Gamma$ are non-increasing, by Proposition 2,

$$
\phi_{\tau} \geq \boldsymbol{\nu} \eta\left(\max _{i \neq j}\left\|x_{i}(\tau)-x_{j}(\tau)\right\|^{2}\right) \geq \boldsymbol{\nu} \eta\left(\Gamma_{\tau}\right) \geq \boldsymbol{\nu} \eta\left(\Gamma_{0}\right) .
$$

Thus,

$$
\int_{0}^{t} \phi_{\tau} d \tau \geq t \boldsymbol{\nu} \eta\left(\Gamma_{0}\right)
$$

and $\Lambda(f(t)) \leq \Lambda_{0} e^{-2 t \boldsymbol{\nu} \eta\left(\Gamma_{0}\right)}$. This shows the convergence to 0 of $\Lambda(t)$. That of $\Gamma(t)$ is similar.

Remark 6 (i) We interpret the convergence of $x(t)$ to a fixed $\boldsymbol{x} \in \Delta_{X}$ as the formation of a tribe and the convergence of $f(t)$ to a fixed $f \in \Delta_{\mathcal{H}}$ as the emergence of a common language as in Examples 2 and 3 of [2]. The first such example is taken from [5] were models are proposed (and studied via simulation) for the origins of language. The second, is a modification of it proposed in [2] for the emergence of common vowel sounds. 
(ii) The assumption of symmetry is plausible in contexts where (unlike the Mother/Baby case discussed in [2, Example 4]) there are no leaders in the liguistic population.

(iii) Detailed learning mechanisms could be introduced by first deriving a result akin to Proposition 4 for discrete time and then follow [2].

(iv) We have not used any argument as those in the proof of Proposition 4. These arguments involved expressions like $\langle x, f\rangle$ which, in the situation at hand, would be meaningless.

\section{References}

[1] F. Cucker and S. Smale. Best choices for regularization parameters in learning theory. Found. Comput. Math., 2:413-428, 2002.

[2] F. Cucker, S. Smale, and D.-X. Zhou. Modeling language evolution. Found. Comput. Math., 4:315-343, 2004.

[3] G. Flierl, D. Grünbaum, S. Levin, and D. Olson. From individuals to aggregations: the interplay between behavior and physics. J. Theor. Biol., 196:397-454, 1999.

[4] A. Jadbabaie, J. Lin, and A.S. Morse. Coordination of groups of mobile autonomous agents using nearest neighbor rules. IEEE Trans. on Autom. Control, 48:988-1001, 2003.

[5] J. Ke, J. Minett, C.-P. Au, and W.S.-Y. Wang. Self-organization and selection in the emergence of vocabulary. Complexity, 7:41-54, 2002.

[6] B. Mohar. The Laplacian spectrum of graphs. In Y. Alavi, G. Chartrand, O.R. Oellermann, and A.J. Schwenk, editors, Graph Theory, Combinatorics, and Applications, volume 2, pages 871-898. John Wiley \& Sons, 1991.

[7] T. Vicsek, A. Czirók, E. Ben-Jacob, and O. Shochet. Novel type of phase transition in a system of self-driven particles. Phys. Rev. Letters, 75:1226-1229, 1995. 